\title{
Traumatismo torácico por arma de fuego
}

\author{
Roberto González L. ${ }^{1,2}$, Alejandra Riquelme U. ${ }^{2}$, Matías Ávalos T. ${ }^{3}$, Rodrigo Reyes M. ${ }^{1,2}$, \\ Enrique Seguel S. ${ }^{1,2}$, Aleck Stockins L. ${ }^{1,2}$, Andrés Jadue T. ${ }^{1}$, Claudio Toloza A. ${ }^{3}$, \\ Felipe Alarcón O. ${ }^{3}$, Sebastián Barra M..$^{3}$ y Emilio Alarcón C. ${ }^{1,2}$
}

\section{Thoracic trauma by firearms}

Background: Thoracic trauma by firearms (TTF) are increasingly frequent. Aim: To describe clinical characteristics, morbidity and mortality and the evolution over time of patients hospitalized due TTF. Materials and Method: Longitudinal analytical study. Period January 1981 - December 2018. Database review, prospective protocols and clinical files. Microsoft Excel ${ }^{\circledR}$ spreadsheet and SPSS24 ${ }^{\circledR}$ program with chi square and Mann-Whitney tests were used. Description of characteristics of TTF in hospitalized patients and comparison of TTF by periods. Trauma severity indexes (TSI) were calculated: Injury Severity Score (ISS), Revised Trauma Score (RTS-T), Trauma Injury Severity Score (TRISS). Results: Total: 4306 hospitalized patients due thoracic trauma (TT), 205 (4.8\%) hospitalized due TTF. Men: 188 (91.7\%), average age $28.8 \pm 11.2$ years, isolated TTF $115(56.1 \%)$, associated with extrathoracic lesions $90(43.9 \%)$, and of these $55(26.8 \%)$ were considered polytraumatism. Mechanisms: aggression $193(94.1 \%)$, self-harm 11 (5.4\%) and accidental 1 (0.5\%). Frequent thoracic injuries and/or findings: hemothorax 127 (62.0\%), pneumothorax $96(46.8 \%)$ and pulmonary contusion $51(24.9 \%)$. Definitive treatment: Pleurotomy 88 (42.9\%), surgery $71(34.6 \%)$ and medical treatment 46 (22.4\%). Median hospitalization 7 days. According TSI: Average ISS $16.7 \pm 11.7$, average RTS-T $11.1 \pm 2.1$, average TRISS 9.6. Morbidity: 44 (21.5\%). Mortality: $14(6.8 \%)$. There is an increase in polytraumatism and average TRISS, without changes in mortality. Discussion: The majority of TTF were isolated TT. Approximately one third of patients required surgery. The observed mortality is lower than expected. Changes in TTF were observed over time.

Key words: Firearms; trauma severity indices; thoracic injuries; penetrating wounds; thoracic surgery.

\section{Resumen}

Introducción: Los traumatismos torácicos por armas de fuego (TTAF) son cada vez más frecuentes. Objetivos: Describir características clínicas, morbilidad, mortalidad y la evolución a través del tiempo de hospitalizados por TTAF. Materiales y Método: Estudio analítico longitudinal. Período enero de 1981diciembre de 2018. Revisión base de datos, protocolos prospectivos y fichas clínicas. Se utilizó planilla Microsoft Excel ${ }^{\mathbb{B}}$ y programa SPSS $24^{\mathbb{B}}$ con chi cuadrado y de Mann-Whitney. Descripción de características de TTAF en pacientes hospitalizados y comparación por períodos. Se calcularon índices de gravedad del traumatismo (IGT): Injury Severity Score (ISS), Revised Trauma Score (RTS-T), Trauma Injury Severity Score (TRISS). Resultados: Total 4.306 pacientes hospitalizados por traumatismo torácico (TT), 205 $(4,8 \%)$ hospitalizados por TTAF. Hombres: $188(91,7 \%)$, edad promedio $28,8 \pm 11,2$ años, TTAF aislado $115(56,1 \%)$, asociado a lesiones extratorácicas $90(43,9 \%)$, de estos $55(26,8 \%)$ se consideraron politraumatismos. Mecanismo: Agresión 193 (94,1\%), autoagresión 11 (5,4\%) y accidental 1 (0,5\%). Lesiones y/o hallazgos torácicos más frecuentes: Hemotórax $127(62,0 \%)$, neumotórax $96(46,8 \%)$ y contusión pulmonar 51 (24,9\%). Tratamiento definitivo: Pleurotomía 88 (42,9\%), cirugía $71(34,6 \%)$ y tratamiento médico 46 (22,4\%). Mediana de hospitalización 7 días. Según IGT: ISS promedio 16,7 $\pm 11,7$, RTS-T promedio 11,1 $\pm 2,1$, TRISS promedio 9,6. Morbilidad: 44 (21,5\%). Mortalidad: 14 (6,8\%). En los diferentes períodos, se observó aumento de politraumatismos y TRISS, sin cambios en mortalidad. Discusión: La mayoría de los TTAF fueron aislados. Aproximadamente un tercio de los pacientes requirió cirugía. La mortalidad observada es menor a la esperada. Se observan cambios en los TTAF a través del tiempo. Palabras clave: armas de fuego; índices de gravedad del trauma; traumatismos torácicos; heridas penetrantes; cirugía torácica. 


\section{Introducción}

El traumatismo torácico (TT) representa cerca del $10 \%$ de todos los traumatismos. Se clasifica en contuso o penetrante, siendo los agentes más frecuentes de este último las armas blancas y de fuego ${ }^{1}$.

El descubrimiento de la pólvora en China en el siglo IX, y su posterior difusión a Medio Oriente y Europa en el siglo XIV, permitió la creación de un nuevo tipo de armamento, revolucionando la industria bélica. Esto trajo como consecuencia nuevos desafíos para la medicina del traumatismo.

Los TT por arma de fuego (TTAF) se definen como todas las lesiones traumáticas causadas por proyectiles lanzados explosivamente, que afectan las paredes torácicas y/o el contenido visceral ubicado entre la base del cuello y el límite inferior de las costillas y diafragma ${ }^{2}$. Los TTAF son cada vez más frecuentes en nuestro medio ${ }^{1}$ y presentan una mortalidad mayor que los TT por arma blanca, debido a la multiplicidad y gravedad de las lesiones torácicas y extratorácicas asociadas, principalmente las abdominales ${ }^{2,3}$.

A pesar de que los TT se encuentran ampliamente descritos en la literatura internacional, es escasa la bibliografía en nuestro medio y más aún respecto a TTAF.

Nuestros objetivos son describir las características clínicas, morbilidad, mortalidad y la evolución a través del tiempo de pacientes hospitalizados por TTAF.

\section{Materiales y Método}

Se realizó un estudio analítico longitudinal de todos los pacientes hospitalizados por TTAF en el Hospital Clínico Regional "Dr. Guillermo Grant Benavente" de Concepción, Chile, en el período comprendido entre enero de 1981 y diciembre de 2018. La información se obtuvo a partir de protocolos prospectivos de TT realizados al ingreso del paciente, revisión de base de datos prospectiva de TT del equipo de Cirugía Cardiotorácica, protocolos quirúrgicos y fichas clínicas. Se ordenaron los datos mediante planilla Microsoft Excel $^{\mathbb{}}$ y se realizó análisis estadístico con programa SPSS $24^{\circledR}$, con función chi cuadrado para variables cualitativas y prueba de Mann-Whitney para variables cuantitativas. Además, de regresión lineal de Pearson para análisis de evolución temporal, calculando $\mathrm{R}^{2}$.

Se describen edad, sexo, características clínicas, mecanismos, tratamiento, estadía hospitalaria, morbilidad, mortalidad e índices de gravedad del traumatismo (IGT) como Injury Severity Score (ISS) que corresponde a un índice basado en lesión anatómica, Revised Trauma Score Triage (RTS-T), el cual se centra en parámetros fisiológicos y Trauma Injurity Severity Score (TRISS), índice mixto que incluye los dos anteriores. Se consideró politraumatizado a aquellos pacientes con ISS $>16$ puntos $^{4,5}$.

Para buscar tendencias de frecuencia se agrupó en cuatro períodos: 1981 a 1990, 1991 a 2000, 2001 a 2010 y 2011 a 2018; y para comparar características se dividió en dos períodos de 1981 a 2010 y 2011 a 2018, dado el aumento en la última década de TTAF.

Se consideró significativo $\mathrm{p}<0,05$.

\section{Resultados}

La totalidad de TT hospitalizados fue de 4.306 pacientes. De ellos 205 (4,8\%) fueron TTAF, 188 $(91,7 \%)$ hombres, con relación hombre/mujer: $11,1 / 1$. La edad promedio fue $28,8 \pm 11,2$ años.

El mecanismo más frecuente fue agresión en 193 $(94,1 \%)$ casos. Las municiones fueron balas en 166 $(81,0 \%)$ y perdigones de escopeta en $39(19,0 \%)$ casos. La mayoría de los TTAF ocurrieron en la vía pública en $168(82,0 \%)$, en domicilio en $18(8,8 \%)$ y en otros sitios como cárceles, estadios y recintos privados en $19(9,3 \%)$ casos. Los TT aislados fueron $115(56,1 \%)$ y con lesiones asociadas $90(43,9 \%)$, de estos se consideraron politraumatizados 55 $(26,8 \%)$ (Tabla 1).

Las lesiones y/o hallazgos torácicos más frecuentes se presentan en la Tabla 2. Las lesiones extratorácicas más frecuentes fueron las abdominales en 44 $(21,5 \%)$ casos.

En cuanto al tratamiento definitivo de la lesión torácica (Tabla 3), se realizó tratamiento invasivo en $159(77,6 \%)$ pacientes, de estos, fueron tratados con pleurotomía exclusiva $88(42,9 \%)$ y con cirugía torácica $71(34,6 \%)$. Los pacientes que recibieron tratamiento médico exclusivo fueron $46(22,4 \%)$.

Se trataron 71 pacientes con cirugía torácica, de estas fueron urgentes $(\leq 4 \mathrm{~h}) 53(74,6 \%)$. Las indicaciones más frecuentes de cirugía fueron los traumatismos penetrantes cardiacos (TPC) en $21(29,6 \%)$ y los hemotórax complicados en 14 (19,7\%). Se realizaron 6 reoperaciones, lo que equivale al 8,5\% de los pacientes que se trataron con cirugía torácica (Tabla 4). Se registraron 52 cirugías extratorácicas (Tabla 5).

Presentaron morbilidad 44 (21,5\%) pacientes. La mortalidad fue de $14(6,8 \%)$ casos, teniendo como causa principal el shock hipovolémico en $6(2,9 \%$ 
Tabla 1. Edad, sexo, mecanismo, munición, sitio y tipo del traumatismo de pacientes hospitalizados por traumatismo torácico por arma de fuego

\begin{tabular}{|lc|}
\hline & Total TTAF (205) \\
\hline Edad (años) & \\
Promedio & $28,8 \pm 11,2$ \\
Mediana & 26 \\
Rango & $11-73$ \\
Adulto mayor ( $\geq 60$ años) & $6(2,9 \%)$ \\
Sexo & \\
Hombre & $188(91,7 \%)$ \\
Mujer & $17(8,3 \%)$ \\
Mecanismo & \\
Agresión & $193(94,1 \%)$ \\
Autoagresión & $11(5,4 \%)$ \\
Accidente doméstico & $1(0,5 \%)$ \\
Munición & \\
Bala & $166(81,0 \%)$ \\
Perdigón de escopeta & $39(19,0 \%)$ \\
Sitio de traumatismo & \\
Vía pública & $168(82,0 \%)$ \\
Domicilio & $18(8,8 \%)$ \\
Otros & $19(9,2 \%)$ \\
Tipo de traumatismo & \\
TT aislado & $115(56,1 \%)$ \\
TT con lesiones asociadas & $90(43,9 \%)$ \\
Politraumatismo & $55(26,8 \%)$ \\
\hline TAF Traumatismo & \\
\hline
\end{tabular}

TTAF: Traumatismo torácico por arma de fuego; TT: Traumatismo torácico.

Tabla 4. Temporalidad, indicaciones quirúrgicas principales y reoperaciones en pacientes tratados con cirugía torácica por traumatismo torácico por arma de fuego

\begin{tabular}{|c|c|}
\hline & $\begin{array}{l}\text { Total cirugías } \\
\text { torácicas ( } 71)\end{array}$ \\
\hline \multicolumn{2}{|l|}{ Temporalidad de la cirugía } \\
\hline Urgente $(\leq 4 \mathrm{~h})$ & $53(74,6 \%)$ \\
\hline Precoz (> 4-24 h) & $3(4,2 \%)$ \\
\hline Diferida $(>24 \mathrm{~h})$ & $15(21,2 \%)$ \\
\hline \multicolumn{2}{|c|}{ Indicaciones quirúrgicas principales } \\
\hline Penetrante cardiaca & $21(29,6 \%)$ \\
\hline Hemotórax complicado & $14(19,7 \%)$ \\
\hline Hemotórax masivo & $9(12,7 \%)$ \\
\hline Lesión parénquima pulmonar & $7 \quad(9,9 \%)$ \\
\hline Lesión diafragma & $7 \quad(9,9 \%)$ \\
\hline Lesión grandes vasos & $6(8,5 \%)$ \\
\hline Otros & $7(9,9 \%)$ \\
\hline Reoperación torácica & $6(8,5 \%)$ \\
\hline
\end{tabular}

Tabla 2. Hallazgos y/o lesiones torácicas y extratorácicas en pacientes hospitalizados por traumatismo torácico por arma de fuego

\section{Total TTAF (205)}

Hallazgos y/o lesiones torácicas

Hemotórax no masivo $127(62,0 \%)$

Neumotórax

Contusión pulmonar $\quad 51(24,9 \%)$

Enfisema subcutáneo $\quad 47(22,9 \%)$

Fractura costal $\quad 36(17,6 \%)$

Lesión parénquima pulmonar $33(16,1 \%)$

Lesión diafragmática $29(14,1 \%)$

Penetrante cardiaca $\quad 21(10,2 \%)$

Hemotórax masivo $12(5,9 \%)$

Lesión de grandes vasos $\quad 10 \quad(4,9 \%)$

Neumotórax abierto $\quad 4 \quad(2,0 \%)$

Fractura esternal $3(1,5 \%)$

Neumotórax hipertensivo $\quad 2(1,0 \%)$

Lesiones extratorácicas

Abdominales $44(21,5 \%)$

Extremidades $\quad 18(8,8 \%)$

Craneoencefálicas $\quad 12 \quad(5,9 \%)$

Partes blandas $\quad 12 \quad(5,9 \%)$

Faciales $\quad 7 \quad(3,4 \%)$

Fractura de pelvis $\quad 1 \quad(0,5 \%)$

TTAF: Traumatismo torácico por arma de fuego.

Tabla 3. Tratamiento definitivo de la lesión torácica realizado en pacientes hospitalizados por traumatismo torácico por arma de fuego

\begin{tabular}{|lc|}
\hline & Total TTAF (205) \\
\hline Tratamiento invasivo & $\mathbf{1 5 9}(\mathbf{7 7 , 6 \% )}$ \\
Pleurotomía exclusiva & $88(42,9 \%)$ \\
Cirugía torácica & $71(34,6 \%)$ \\
Médico exclusivo & $\mathbf{4 6}(\mathbf{2 2 , 4 \% )}$ \\
\hline
\end{tabular}

TTAF: Traumatismo torácico por arma de fuego.

Tabla 5. Cirugías extratorácicas realizadas en pacientes con traumatismo torácico por arma de fuego

\begin{tabular}{|lc|}
\hline & $\begin{array}{c}\text { Total cirugías } \\
\text { extratorácicas (52) }\end{array}$ \\
\hline Abdominales & $38(73,1 \%)$ \\
Traumatológicas & $5(9,6 \%)$ \\
\hline Vasculares & $4(7,7 \%)$ \\
\hline Partes blandas & $4(7,7 \%)$ \\
Craneoencefálica & $1(1,9 \%)$ \\
Reoperación extratorácica & $6(11,5 \%)$ \\
\hline
\end{tabular}


Tabla 6. Morbilidad, mortalidad, estadía hospitalaria e índices de gravedad del traumatismo en pacientes hospitalizados por traumatismo torácico por arma de fuego

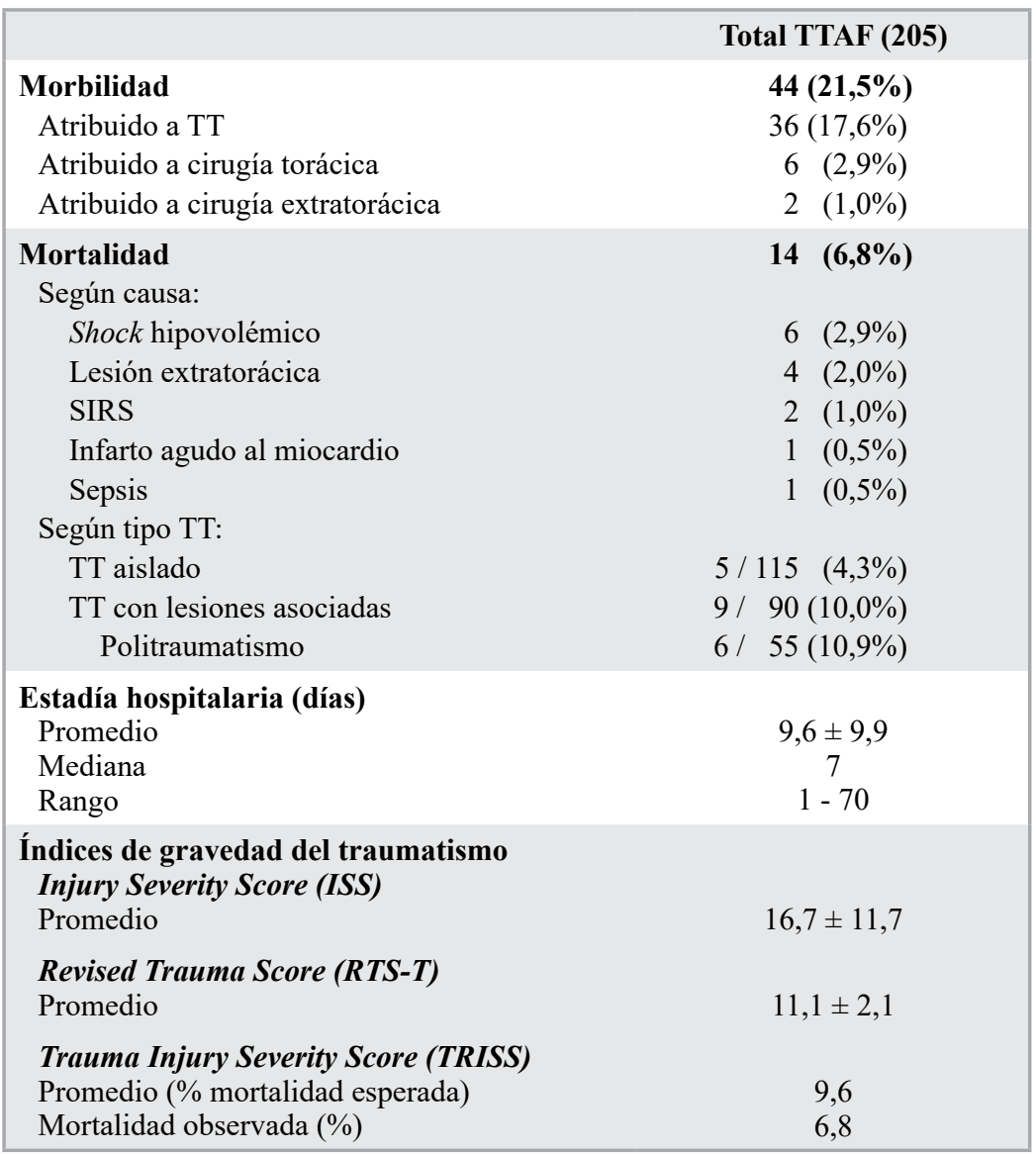

TTAF: Traumatismo torácico por arma de fuego; TT: Traumatismo torácico; SIRS: Síndrome repuesta inflamatoria sistémica.

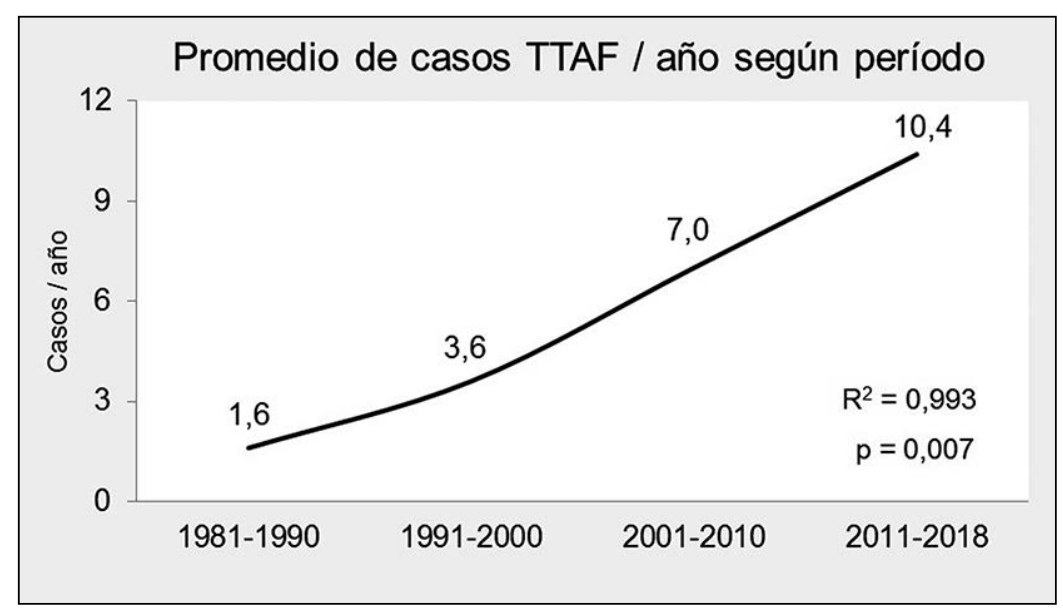

Figura 1. Promedio de casos de traumatismos torácicos por arma de fuego por año según período. del total de TTAF y el $42,8 \%$ de los fallecidos). De los pacientes que fallecieron, $11(78,6 \%)$ fueron durante la primera semana y $3(21,4 \%)$ entre 8 y 30 días posterior al traumatismo. La mediana de hospitalización fue 7 días (Tabla 6).

$\mathrm{Al}$ analizar la evolución temporal, se observa un aumento en el promedio de 1,6 a 10,4 de TTAF por año (Figura 1), presentándose en el último año 17 casos. Existe un alza en el número absoluto de los TTAF, así como su proporción respecto de los TT totales, los penetrantes y los por agresión, todos con significancia estadística (Figura 2).

Al comparar las características (Tabla 7), existe un aumento en los politraumatismos y de la estadía hospitalaria. Se observa un aumento en el ISS promedio, de politraumatismos graves (ISS $\geq 25$ ) y TRISS, además, de una disminución en el porcentaje de cirugías y morbilidad, todo con diferencias estadísticamente significativas. La mortalidad observada no presenta cambios estadísticamente significativos.

\section{Discusión}

A lo largo del tiempo el tratamiento de las heridas por arma de fuego (HAF) ha variado enormemente. En 1812 el doctor M. Richerand publicó en The New England Journal of Medicine and Surgery and The Collateral Branches of Science un artículo dedicado a las HAF, donde describe como primera indicación del tratamiento la extracción del proyectil, y que "nada puede contraindicar su búsqueda". En ese entonces prácticamente no se describen heridas torácicas por armas de fuego, probablemente debido a que las lesiones eran muy leves o mortales?

Así como ha variado su tratamiento, también ha variado la frecuencia de este tipo de lesiones. En un artículo recientemente publicado por nuestro grupo se evidencia que los TTAF se han vuelto cada vez más frecuentes en nuestro medio ${ }^{1}$.

El arma de fuego puede definirse como una máquina termodinámica capaz de transformar energía química en energía cinética con el objetivo de lanzar violentamente proyectiles aprovechando la fuerza expansiva de los gases que se producen en su interior por deflagración de la pólvora ${ }^{8}$. El calificativo "de fuego" se atribuye a la llamarada emitida por la boquilla del arma.

Existen diferentes formas de clasificar las armas de fuego. Según tamaño se dividen en armas de fuego pesada o de arrastre y en armas ligeras o portátiles. Las armas de fuego pesadas se caracterizan por su munición de gran calibre, representadas principalmente por artillería, que producen, entre 


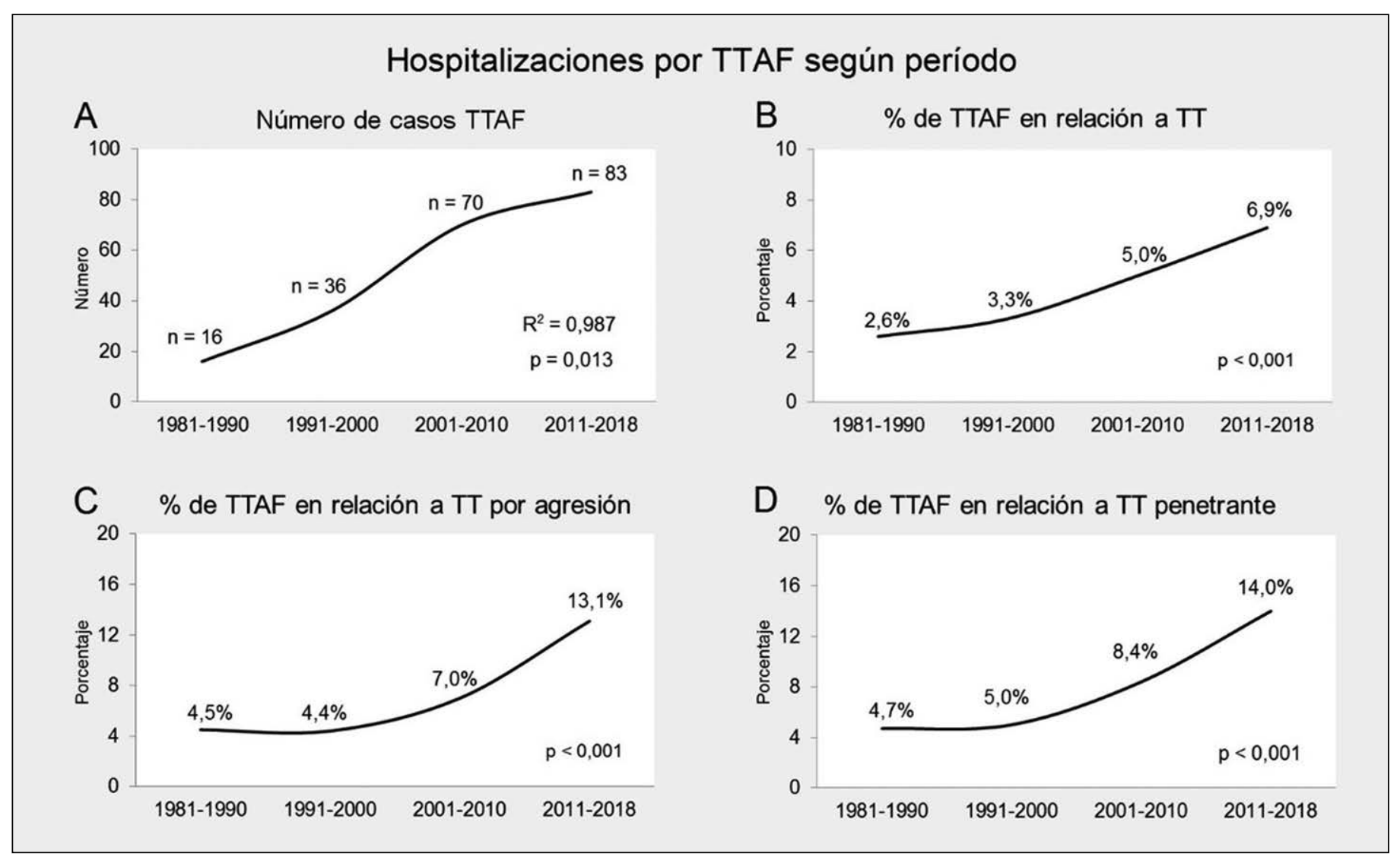

Figura 2. Número de casos traumatismos torácicos por arma de fuego y porcentaje en relación a traumatismos torácicos totales, por agresión y penetrantes según período.

Tabla 7. Comparación entre períodos de características de los traumatismos torácicos por arma de fuego

\begin{tabular}{|c|c|c|c|}
\hline & $\begin{array}{c}\text { Período 1981-2010 } \\
\text { (122) }\end{array}$ & $\begin{array}{c}\text { Período 2011-2018 } \\
\text { (83) }\end{array}$ & $\mathbf{p}$ \\
\hline TTAF aislado & $87(71,3 \%)$ & $28(33,7 \%)$ & $<0,001 *$ \\
\hline Cirugía torácica & $50(41,0 \%)$ & $21(25,3 \%)$ & $0,021 *$ \\
\hline Morbilidad & $32(26,2 \%)$ & $12(14,5 \%)$ & $0,044 *$ \\
\hline Estadía hospitalaria (días) ${ }^{* * *}$ & 7,9 & 11,9 & $0,002 *$ \\
\hline Injury Severity Score (ISS) $\geq 25$ & $28(23,0 \%)$ & $32(38,6 \%)$ & $0,016^{*}$ \\
\hline Injury Severity Score (ISS)*** & $13,9 \pm 9,4$ & $20,8 \pm 13,4$ & $<0,001 *$ \\
\hline
\end{tabular}

TTAF: Traumatismo torácico por arma de fuego. *Estadísticamente significativo. **En el grupo con TT aislado no hay mortalidad durante el segundo período y en los pacientes con politraumatismo la mortalidad disminuye a aproximadamente la mitad. $* * *$ Promedio. 
otras, lesiones por esquirlas (lesión por metralla). Las armas ligeras o portátiles se caracterizan por ser transportables por el tirador y poseer un calibre inferior a $20 \mathrm{~mm}$. Estas últimas a su vez, se clasifican según el largo del cañón en armas largas y cortas 9 .

Según la velocidad que adquieren los proyectiles, las armas de fuego se dividen en armas de baja velocidad que impulsan proyectiles a menos de $350 \mathrm{~m} /$ seg (pistolas y revólveres), armas de alta velocidad a más de $600 \mathrm{~m} / \mathrm{seg}$ (rifles automáticos y semiautomáticos) y el grupo intermedio (entre 350-600 m/ seg) que incluye las escopetas ${ }^{10,11}$

En las HAF se pueden distinguir 3 componentes: orificio de entrada, trayectoria y orificio de salida. El primero se produce por el impacto del proyectil a través de la dermis, que es un tejido elástico, por lo que se retrae posterior al paso de la munición, resultando un orificio de menor diámetro que el proyectil $^{10,11}$. En cuanto a la trayectoria esta puede ser recta o desviarse al chocar con huesos, cartílagos, tendones o aponeurosis, siguiendo el plano que ofrezca menor resistencia ${ }^{7,10,11}$. Por lo tanto, se desprende que no siempre el orificio de entrada se encontrará alineado con el de salida, pudiendo este último no existir. En el caso de no encontrar un orificio de salida se debe sospechar que la bala se encuentra intracorpórea.

Gran parte del daño producido es adjudicado a la onda expansiva que ocurre como consecuencia de la energía cinética liberada por el proyectil. Esta energía produce una onda de choque, que desplaza brevemente los tejidos circundantes, formando una cavidad temporal. Al disiparse la energía, los tejidos vuelven a su posición original, a menos que, la onda expansiva supere la resistencia de los tejidos, lo que ocasionaría que estos se desgarren, quedando una cavidad definitiva de mayor diámetro que el proyectil. Es así como en el orificio de salida pueden encontrarse restos de órganos lesionados en el trayecto ${ }^{10}$.

Las HAF producto de perdigones son complejas, ya que cada uno de los perdigones tendrá un orificio de entrada y trayecto distinto. El grado de dispersión de las municiones dependerá de la distancia a la cual se disparó. Cuando la distancia es corta la dispersión es menor, el conjunto se traslada como una sola masa con mayor energía cinética, produciendo mayor daño tisular y produciéndose en este tipo de lesiones mayor mortalidad ${ }^{10-12}$.

Particularmente, los TTAF presentan una elevada mortalidad, debido a que el tórax contiene estructuras como el corazón y grandes vasos. Las lesiones cardiacas producto de proyectiles constituyen generalmente una urgencia, presentando elevada mortalidad en el sitio del suceso, consultando en las unidades de urgencia pacientes que no siempre tienen deterioro hemodinámico ${ }^{13,14}$. Cabe destacar, que esta serie está compuesta exclusivamente por pacientes hospitalizados por lo que no representa el total de TTAF, ya que muchos de estos pacientes fallecen en el lugar del traumatismo o no alcanzan a recibir atención hospitalaria.

En diferentes estudios de varias regiones se describe que la mayoría de los traumatismos por arma de fuego, al igual que los TT penetrantes, ocurren principalmente en hombres jóvenes a diferencia de los adultos mayores donde predominan los contusos $^{1,13,15-20}$. En nuestra serie los resultados son similares, donde un $91,7 \%$ es hombre, con una edad promedio de 28,8 años.

El mecanismo predominante es la agresión $(94,1 \%)$, seguido de autoagresión $(5,4 \%)$ y solo un caso accidental $(0,5 \%)$. Existe poca literatura sobre el mecanismo de los TTAF, sin embargo, sí hay información disponible acerca de los TT penetrantes. De los existentes, los resultados parecen variar según su localización geográfica, por ejemplo, estudios europeos sobre traumatismos por armas de fuego reportan cifras distintas a las aquí expuestas, con menores porcentajes de agresión, mientras que otros estudios latinoamericanos describen en casi su totalidad mecanismos relacionados con violen$\operatorname{cia}^{13,15,21-23}$.

La mayoría de los TTAF atendidos en nuestro hospital fueron por balas $(81,0 \%)$, siendo menor el porcentaje de los ocurridos por perdigones de escopeta $(19,0 \%)$. Los diferentes agentes varían según el contexto en el cual se desarrollan los distintos estudios, por ejemplo, en estudios en zonas de guerra se encuentran lesiones por metralla y proyectiles de alta velocidad, poco comunes en zonas urbanas. En los pocos estudios donde se describe los TTAF por perdigones de escopeta corresponden al 16,3\% de los pacientes ${ }^{24}$. Cabe destacar que en nuestra serie solo se reportan casos de violencia civil, por lo que se asume que no se tratan de proyectiles de alta velocidad.

Un estudio de 755 pacientes con TT penetrante describe un 53,0\% de lesión torácica aislada, coincidiendo con nuestros resultados ${ }^{17}$. Los TT con lesiones asociadas, y sobre todo los politraumatismos, tienen en general una mayor mortalidad, sin embargo, los traumatismos aislados también pueden presentar elevada mortalidad debido a lesiones como los TPC y lesiones de grandes vasos ${ }^{25}$.

Los hallazgos y/o lesiones torácicas más frecuentes fueron el hemotórax no masivo, neumo- 
tórax, contusión pulmonar y enfisema subcutáneo. Esto se condice con la literatura donde las lesiones pulmonares son las más frecuentes ${ }^{3,17,26}$. Cabe mencionar que los TPC corresponden a un 10,2\% de los TTAF y que estos forman parte de una entidad distinta, con características propias y mayor mortalidad $^{25}$.

En cuanto a las lesiones extratorácicas, observamos que las más frecuentemente asociadas a los TTAF son las abdominales ${ }^{2}$, en discordancia con lo que ocurre en los TT en general, donde son las craneoencefálicas y de extremidades ${ }^{1}$. Esto explica, además, el porcentaje de lesiones diafragmáticas $(14,1 \%)$ que se observa en nuestra serie.

Se requirió de tratamiento invasivo en la mayoría de los pacientes $(77,6 \%)$, correspondiendo en $\mathrm{su}$ mayoría a pleurotomía (42,9\%). Series europeas describen tratamiento suficiente con pleurotomía en el 76,3\% de los $\operatorname{casos}^{22-27}$. La necesidad de cirugía torácica descrita en distintas revisiones oscila entre $5,8 \%-71,0 \%{ }^{17,28}$, esto se puede deber a las diferencias epidemiológicas en las distintas poblaciones, además, la mayoría de los estudios incluyen grupos heterogéneos de lesiones como las toracoabdominales. En los pacientes ingresados en nuestro hospital, la necesidad de cirugía fue de un $34,6 \%$, siendo la mayoría $(74,6 \%)$ de forma urgente ( $\leq 4 \mathrm{~h}$ ). La urgencia se explica posiblemente por las presentaciones graves, encontrándose lesiones que incluyen TPC, hemotórax masivos y lesiones de grandes vasos, las que requieren exploración y resolución quirúrgica inmediata. Por otra parte, las cirugías diferidas correspondieron a un $21,2 \%$ y se realizaron principalmente debido a complicaciones del traumatismo, dentro de ellas el hemotórax complicado (retenido, coagulado y/o infectado).

Distintas series reportan morbilidad del TTAF entre $16,3 \%-23,3 \%{ }^{16,24}$. En nuestra serie un $21,5 \%$ presentó morbilidad, atribuyéndose esta en su mayoría al traumatismo, siendo mucho menos frecuentes las atribuidas a las cirugías.

La mortalidad en pacientes hospitalizados con TTAF oscila entre 4,5\%-28,0\% ${ }^{3,17,24}$. En nuestra serie fallecieron $14(6,8 \%)$ pacientes, siendo la principal causa el shock hipovolémico y ocurriendo la gran mayoría $(78,6 \%)$ durante la primera semana. Los pacientes que fallecieron posterior a la primera semana fueron $3(21,4 \%)$, donde las causas las constituyen el síndrome de respuesta inflamatoria sistémica y la sepsis. Se observa que los politraumatismos presentan mayor mortalidad.

Los IGT son scores que orientan sobre la gravedad y mortalidad esperada del traumatismo, permitiendo estandarizar y comparar distintos estudios ${ }^{4,5}$. En nuestro medio, observamos que la mortalidad observada es menor a la esperada según IGT, probablemente por la mejoría en las técnicas diagnósticas y tratamiento precoz del traumatismo por equipos entrenados ${ }^{1}$.

Finalmente, al analizar la evolución a través del tiempo de los TTAF se observa un incremento del número absoluto y en proporción a los traumatismos torácicos, como también el de casos por año. Cuando se compara con el último período existe un aumento de los politraumatismos, politraumatismos graves (ISS $\geq 25$ ) y de la estadía hospitalaria. Por el contrario, la morbilidad y la necesidad de cirugía torácica disminuyen al comparar ambos períodos. La mortalidad observada disminuye (no significativamente) a pesar de aumentar significativamente la gravedad de los pacientes, lo que se grafica en un aumento significativo del TRISS (mortalidad esperada).

En conclusión, en nuestro hospital existe un aumento significativo del número de TTAF. Este se presenta frecuentemente en hombres jóvenes. Ocurren en su mayoría en la vía pública y producto de agresiones. Las lesiones y/o hallazgos más frecuentes son el hemotórax y neumotórax, mientras que las lesiones extratorácicas asociadas predominantes fueron las abdominales. La mayoría de los pacientes requirieron tratamiento invasivo y aproximadamente un tercio requirió cirugía torácica. Actualmente existe una mayor proporción de politraumatismos y mayor gravedad de los pacientes, no existiendo cambios significativos en la mortalidad.

\section{Responsabilidades éticas}

Protección de personas y animales. Los autores declaran que para esta investigación no se han realizado experimentos en seres humanos ni en animales.

Confidencialidad de los datos. Los autores declaran que en este artículo no aparecen datos de pacientes.

Conflictos de interés: no hay. 


\section{Referencias}

1. González R, Riquelme A, Fuentes A, Saldías R, Reyes R, Seguel E, et al. Traumatismo torácico: caracterización de hospitalizaciones durante tres décadas. Rev Med Chile 2018;146:196-205.

2. Duhamel P, Bonnet PM, Pons F, Jourdan $P$, Jancovici R. Thoracic ballistic traumatisms. Wounding agents and wound ballistic. Ann Chir Plast Esthet. 2003;48:128-34.

3. Madiba TE, Thomson SR, Mdlalose N. Penetrating chest injuries in the firearm era. Injury 2001;32:13-6.

4. Keel M, Trenz O. Pathophysiology of polytrauma. Injury Int J Care Injured 2005;36:691-709.

5. Mica L, Rufibach K, Keel M, Trentz O. The risk of early mortality of polytrauma patients associated to ISS, NISS, APACHE II values and prothrombin time. J Trauma Manag Outcomes 2013;7:6. doi: 10.1186/1752-2897-7-6.

6. Richerand M. On gun-shot wounds. N Engl J Med. 1812;1:344-58.

7. Espinoza R. Heridas por arma de fuego: ¿Qué ha cambiado? Rev Chil Cir. 2000;52:425-30.

8. Luque J. Penetrating wounds firearms to the central nervous system: part one: historical background and ballistics. Rev Fac Med. 2007;15:134-8.

9. Wallace J, editor, Chemical analysis of firearms, ammunition, and gunshot residue, Boca Raton: CRC Press; 2008.

10. García G, Deichler F, Torres E. Lesiones por armas de fuego desde la perspectiva medico-criminalística. Rev Chil Cir. 2011; 63:327-31
11. Stefanopoulos $P$, Hadjigeorgiou G, Filippakis K, Gyftokostas D. Gunshot wounds: A review of ballistics related to penetrating trauma. J Acute Dis. 2014;3:178-85.

12. Ordog G, Wasserberger J, Balasubramanium S. Shotgun wound ballistics. J Trauma 1988;28:624-31.

13. Mäkitie I, Pihlajamäki H. Fatal firearm injuries in Finland: a nationwide survey. Scand J Surg. 2002;91:328-31.

14. Saldías R, González R, Núñez E, Alarcón E, Santander C, Seguel E, et al. Proyectiles cardíacos. Rev Chil Cir. 2003; 55:70-4.

15. The Global Burden of Disease 2016 Injury Collaborators. Global mortality from firearms, 1990-2016. JAMA 2018;320:792-814.

16. Störmann $\mathrm{P}$, Gartner $\mathrm{K}$, Wyen $\mathrm{H}$, Lustenberger T, Marzi I, Wutzler S. Epidemiology and outcome of penetrating injuries in a Western European urban region. Eur J Trauma Emerg Surg. 2016;42:663-9.

17. Inci I, Ozçelik C, Taçyildiz I, Nizam O, Eren N, Ozgen G. Penetrating chest injuries: unusually high incidence of high-velocity gunshot wounds in civilians practice. World $\mathrm{J}$ Surg. 1998;22:438-42.

18. Biasutto S, Moral A, Bella J. Firearmrelated injuries: clinical considerations on 1326 cases. Int Surg. 2006;91:39-43.

19. González R, Riquelme A, Toloza C, Reyes R, Seguel E, Stockins A, et al. Traumatismo torácico contuso. Rev Chil Enferm Respir. 2019;35:96-103.

20. González R, Fuentes A, Riquelme A, Reyes R, Seguel E, Stockins A, et al.
Traumatismo torácico en el adulto mayor. Rev Cir. 2020;72:224-30.

21. Ruiz J, Sanchez-Palencia A, Cueto A, Díez J. Thoracic wounds: review of 90 cases. Arch Bronconeumol. 1999;35:8490.

22. González R, Riquelme A, Toloza C, Reyes R, Seguel E, Stockins A, et al. Traumatismo torácico por arma blanca. Rev Cir. 2020;72:137-43.

23. Díaz J, Enríquez L, Arriaga J, Gutiérrez P. Trauma penetrante en abdomen y tórax: Estudio de casos en el Hospital General de Ciudad Juárez. Cir Gen. 2009;31:9-13.

24. Eren S, Balci A, Ulku R, Cakir O, Eren $\mathrm{M}$. Thoracic firearm injuries in children: management and analysis of prognostic factors. Eur J Cardiothorac Surg. 2003;23:888-93.

25. González R, Riquelme A, Fuentes A, Canales J, Seguel E, Stockins A, et al. Traumatismo penetrante cardiaco: caracterización, resultados inmediatos y variables asociadas a morbilidad y mortalidad en pacientes operados. Rev Cir. 2019;71:245-52.

26. Melo A, Moreira L, Pessoa F, SaintMartin N, Ancilotti R, Souza A, et al. Tomographic aspects of penetrating thoracic trauma: injuries from firearms and other weapons. Radiol Bras. 2017;50:372-7.

27. Kristek J, Krajinovic Z, Horvat V, Fijacko $\mathrm{V}$. Lung lesions in penetrating war injuries of the chest. Acta Med Croatica 2006;60:375-7.

28. Sett S, Busse E, Boyd T, Burgess J. Penetrating chest wounds: a 10-year review. Can J Surg. 1987;30:371-3. 